\section{Decidua-induced impediments}

Pregnancy represents an allogeneic immune challenge for mothers and their developing embryos, yet most mothers do not reject their offspring in utero. In Science, Erlebacher and colleagues show that entry of cells of the immune system is blocked at the site of embryo implantation. Decidual stromal tissues, which form the specialized interface that separates maternal tissues from fetal tissues, lack expression of inflammatory chemokines such as CXCL9, CXCL10 and CCL5, which prevents the recruitment of activated $\mathrm{T}$ cells, including those specific for fetal antigen. The differentiation of endometrial stromal cells into decidual stromal cells during pregnancy is accompanied by the trimethylation of histone $\mathrm{H} 3$ Lys27 at the promoters of $\mathrm{Cxcl9}, \mathrm{Cxcl} 10$ and $\mathrm{Ccl} 5$, which suggests that active repression occurs at those loci. The factors responsible for specific targeting of those loci and how they are induced during fetal implantation remain unknown. Suppressing the expression of inflammatory chemokines provides another layer of immunosuppression that protects embryos from attack by cells of the maternal immune system.

$L A D$

Science 336, 1317-1321 (2012)

\section{To starve, to kill}

Autophagy, a process that results in the degradation of intracellular proteins, is initiated by amino-acid starvation but is also negatively regulated by the metabolic-checkpoint kinase mTOR. In Cell Host and Microbe, Giradin and colleagues examine the initiation and control of xenophagy, a specialized form of autophagy used for the destruction of intracellular pathogens. Epithelial cells infected by the pathogenic bacterium Shigella trigger endomembrane damage, which results in a general amino-acid-starvation response and consequently lower activity of mTOR-a 'double-whammy' that initiates xenophagy and potentially control of this pathogen. In contrast, infection by Salmonella is characterized by only transient disruption of endomembrane integrity and consequent rapid normalization of amino acid concentrations and mTOR activity. By minimally perturbing these stress-related pathways, Salmonella is therefore able to circumvent this cell-intrinsic defense pathway.

Cell Host Microbe (14 June 2012) doi:10.1016/j.chom.2012.04.012

\section{Shaping drug hypersensitivity}

Hypersensitivity is a major complication of certain drug treatments. In the Proceedings of the National Academy of Sciences, Peters et al. shine light on the mechanistic basis of hypersensitivity responses triggered by the antiviral drug abacavir. Some patients treated with abacavir go on to develop sensitivity reactions, but only in the presence of an allele encoding

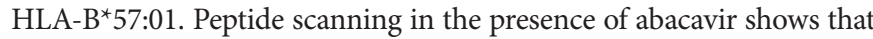
the drug enhances the affinity of certain peptides, especially those with small amino acids such as valine or isoleucine at the carboxy terminus. Structural analysis finds abacavir nestled in the F-pocket binding groove of HLA-B ${ }^{\star} 57: 01$, where it is able to exclude certain peptides yet accept others. Peptide elution of abacavir-bound HLA-B ${ }^{\star}$ 57:01 demonstrates skewing of the peptide repertoire and in effect generates an allogenic antigen complex. Finally, T cells from people sensitized to abacavir show a bias of memory responses to peptides with a carboxy-terminal valine, which supports the structural and biochemical findings.

Proc. Natl. Acad. Sci. USA (19 June 2012) doi:10.1073/pnas.1207934109

\section{Immune calibration by commensals}

Alterations in the intestinal microflora have been associated with susceptibility to metabolic and chronic inflammatory diseases or infections in the intestinal microenvironment. In Immunity, Abt et al. show that alterations in the commensal bacteria also lead to defective systemic antiviral immune responses. In several models of non-gastrointestinal viral infection, depletion of the intestinal flora with antibiotics leads to diminished cellular and humoral immune responses, delayed viral clearance and greater pathology. Equal numbers of innate immune cells are recruited to sites of infection, but macrophages from antibiotic-treated mice have lower expression of genes encoding molecules involved in antiviral defense and interferon-responsive pathways, such as Mx1, Stat1, Irf7, Ifit3 and Ifnb at steady state, and show impaired phosphorylation of STAT1 during infection. These results suggest that commensal bacteria maintain the optimal responsiveness of macrophages to stimulation with interferon, which in turn affects the kinetics and amplitude of the immune response. Immunity (14 June 2012) doi:10.1016/j.immuni.2012.04.011

\section{Local suppressor of inflammation}

Diet can influence the composition and complexity of the gut microbiome in a host. In Nature, Devkota et al. report that diets rich in milk fat skew the microbiome of mice and enhance the propensity of susceptible mice to develop colitis. Milk fat increases taurine conjugation of bile acids, which then promote growth of the sulfite-reducing bacteria Bilophila wadsworthia, an otherwise rare occupant of the microbiome. Germ-free mice can be monoclonized by $B$. wadsworthia only in the presence of milk fat or taurine but not by other diets. This bacterial colonization leads to higher expression of interleukin 12 (IL-12) and interferon- $\gamma$ by mesenteric lymph node cells in an as-yet-unknown way. Although wild-type mice do not develop immunopathology, those that lack IL-10 expression are more prone to disease. Thus, diet can represent a major contributing factor to inflammatory bowel disease in genetically susceptibile people. $L A D$ Nature (13 June 2012) doi:10.1038/nature11225

Written by Laurie A. Dempsey, Zoltan Fehervari \& Ioana Visan
Several microRNAs are known to affect the function and development of lymphocytes and to modulate autoimmune pathogenesis. In Nature Medicine, Zhu et al. show that expression of the microRNA miR-23b is downregulated at local sites of inflammation in patients with rheumatoid arthritis or systemic lupus erythematosus and in the corresponding mouse models and that this contributes to enhanced pathogenesis. IL-17 suppresses the transcription of miR-23b, partially through the recruitment of the regulatory factors NF- $\mathrm{NB}$ and $\mathrm{I} \kappa \mathrm{B} \zeta$ to the promoter of miR-23b. This microRNA suppresses tumor necrosis factor-, IL-1 $\beta$ and IL-17-mediated activation of NF- $\mathrm{KB}$ by targeting the expression of TAB2 and TAB3, two essential signaling mediators downstream of those proinflammatory cytokines. The expression of miR-23b in nonhematopoietic, radioresistant cells is protective in mouse models of rheumatoid arthritis, systemic lupus erythematosus and multiple sclerosis, which suggests that miR-23b functions as a local anti-inflammatory modulator in many autoimmune diseases.

Nat. Med. (3 June 2012) doi:10.1038/nm.2815 